\title{
A case report on uterus didelphys with unilateral renal agenesis and crouzon syndrome
}

\section{Lavanya Kumari.K., Sravani Chithra Cheruvu*}

Department of Obstetrics and Gynaecology, Rajah Muthaiah Medical College and Hospital, Annamalai University, Chidhambaram, Tamilnadu, India

Received: 4 April 2015

Accepted: 6 June 2015

\section{*Correspondence:}

Dr. Sravani Chithra Cheruvu,

E-mail: drsravanichithra@yahoo.com

Copyright: () the author(s), publisher and licensee Medip Academy. This is an open-access article distributed under the terms of the Creative Commons Attribution Non-Commercial License, which permits unrestricted non-commercial use, distribution, and reproduction in any medium, provided the original work is properly cited.

\begin{abstract}
Uterus didelphys is a type of Müllerian duct anomaly where there is complete duplication of uterine horns and the cervix, with no communication between them, which can be associated with abnormalities of the renal system as seen in this case of a 17 year girl, who attained menarche at 13 years of age and had regular cycles presented with dysmenorrhea. Examination and imaging showed right ovarian cystic mass. Incidentally left unilateral solitary kidney was found. Physical examination showed features of Crouzon Syndrome which is an autosomal dominant genetic disorder also known as Branchial Arch Syndrome. She was admitted for treatment of the symptomatic right adnexal mass. Laparotomy was done. Findings were, a dense adherent mass of $6 \times 6 \mathrm{~cm}$ in right iliac fossa, uterus didelphys with left uterine horn, normal left fallopian tube and ovary, right side hematosalphinx adherent to enlarged right uterine horn of 10 weeks size and right side chocolate cyst. Appendix was elongated with faecolith. Adhesiolysis with removal of right non communicating horn with fallopian tube and ovary and appendicectomy was done.
\end{abstract}

Keywords: Chocolate cyst, Crouzon, Didelphys, Kidney

\section{INTRODUCTION}

A uterus didelphys is a type of Müllerian duct anomaly (class III) where there is complete duplication of uterine horns as well as duplication of the cervix, with no communication between them. ${ }^{1,2}$ It accounts for $8 \%$ of Mullerian anomalies. It is frequently associated with abnormalities of the renal system. Workup for these patients includes ultrasonography and/or hysterosalpingography. If these investigations are inconclusive then MRI and IVP need to be done. Crouzon Syndrome $^{3}$ is an autosomal dominant genetic disorder also known as Branchial arch syndrome. The characteristic features include craniosynostosis, hypertelorism, exophthalmos, beak shaped nose, hypoplastic maxilla. It is associated with complications like arrested brain growth and optic nerve atrophy.

\section{CASE REPORT}

17 year old Miss X, who had regular menstrual cycles since age 13, came with complaints of right sided lower abdominal pain and burning micturition for 4 days. Pain was colicky in nature, aggravated during menstruation. History of dysmenorrhea was present during each cycle, relieved after menstruation. She had two episodes of vomiting.

Examination: Her general condition was fair, vitals were stable. She had hypertelorism, exophthalmos, short upper lip and protruding lower lip. Cardiovascular system, 
respiratory system and central nervous system were within normal limits. On per abdomen examination she had a mass of size $4 \times 2 \mathrm{~cm}$ in the right iliac fossa. No warmth, tenderness was present. Surface was smooth, firm in consistency and margins well defined. On per rectal examination uterus normal size, mass felt.

Investigations: Haemoglobin was $9.2 \mathrm{~g} \%$, random blood sugar $134 \mathrm{mg} / \mathrm{dl}$ for which GTT was done and was within normal limits. Her renal function test, serum electrolytes were within normal limits. Ca125 was 640.9 U/ml. ECG and CXR within normal limits. CT abdomen showed complex right ovarian cystic mass, right ovary not visualised, left unilateral solitary kidney seen.

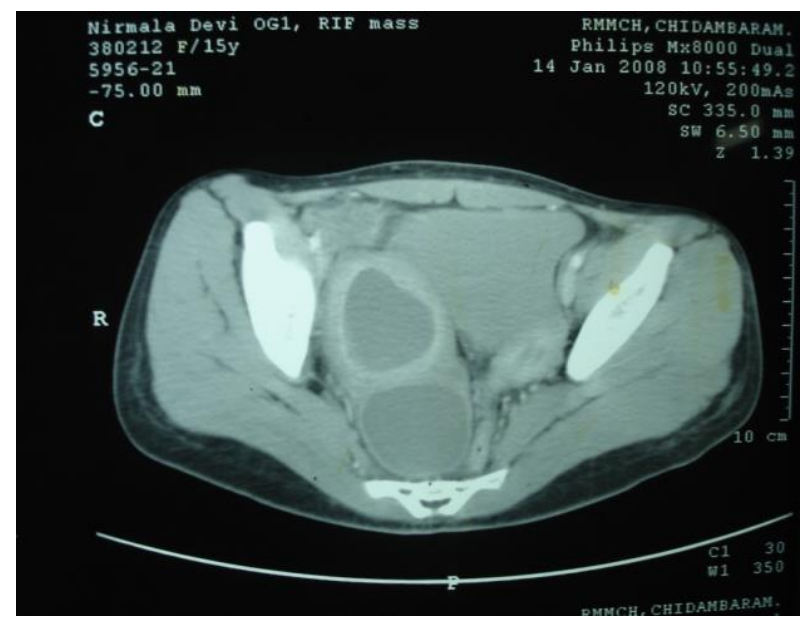

Intravenous pyelogram showed normal functioning left kidney with grade 1 hydronephrosis, non-visualisation of right kidney, pelvic mass causing impression on right bladder wall and spina bifida of LS spine.

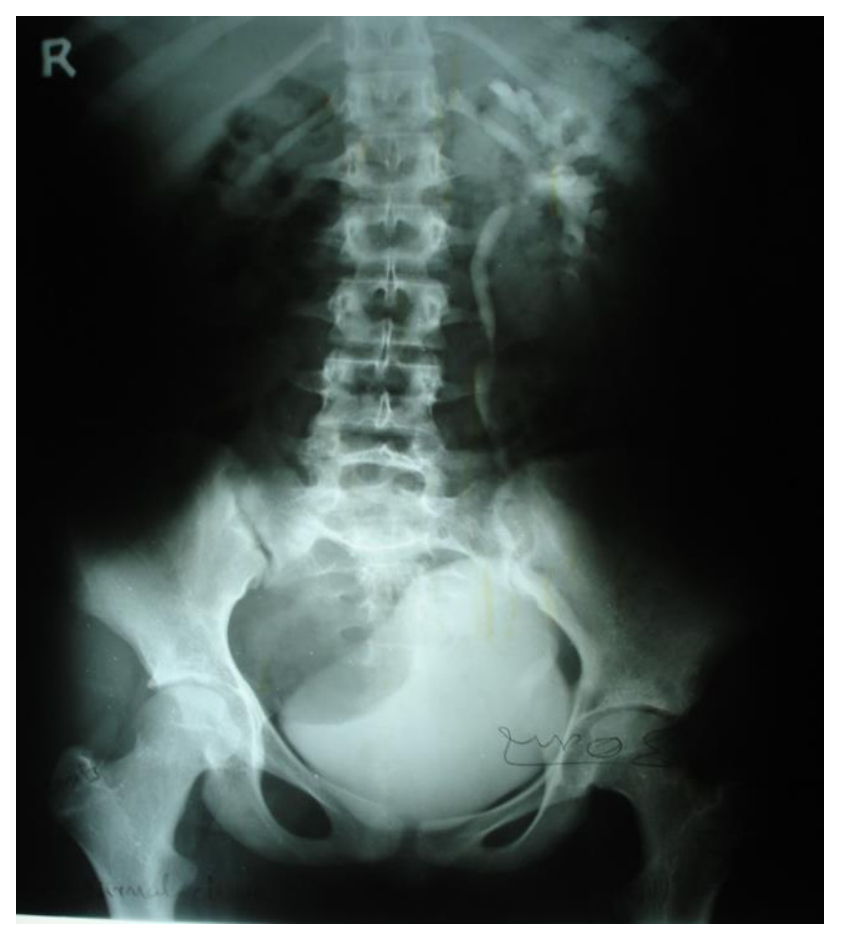

Laparotomy was done. Findings were, a dense adherent mass of $6 \times 6 \mathrm{~cm}$ in right iliac fossa, uterus didelphys with left uterine horn, normal left fallopian tube and ovary, right side hematosalpinx adherent to enlarged right uterine horn of 10 weeks size and right side chocolate cyst. Appendix was elongated with faecolith.

Adhesiolysis with removal of right non communicating horn with fallopian tube and ovary and appendectomy was done. Cyst could not be removed due to adhesions, hence it was opened, and chocolate coloured fluids drained and wash given.

\section{DISCUSSION}

There is a high association between mullerian ${ }^{4}$ duct and renal anomalies such as unilateral renal agenesis. Imaging helps detect, diagnose and distinguish surgically correctable forms of mullerian ${ }^{3}$ malformations from inoperable forms. Abdominal or transvaginal ultrasound is cheap and non-invasive. Three-dimensional (3D) has higher sensitivity and specificity to evaluate malformations. While Computed tomography (CT) has a limited role in the evaluation of female pelvis, magnetic resonance imaging (MRI) is highly sensitive. The diagnosis of a rare mullerian anomaly, known as HerlynWerner-Wunderlich Syndrome (HWWS) should be suspected in women who have ipsilateral renal agenesis with a pelvic mass. Here the patient will present with with signs and symptoms of an acute abdomen with uterus didelphys, unilateral hematocolpos, blind hemivagina, ipsilateral renal agenesis ${ }^{5}$ and menses. In this patient there was no blind hemivagina. ${ }^{2}$ She was counselled and explained about the chance of successful pregnancy in future and the associated complications like infertility, recurrent abortions, preterm labour, intrauterine growth restriction, malpresentations and increased rate of caesarean sections.

Funding: No funding sources

Conflict of interest: None declared

Ethical approval: Not required

\section{REFERENCES}

1. Wahi M, Trivedi P. Unusual Laparoscopy Finding with Previous Laparotomy for Endometrioma: A Rare Case Report. J Gynecol Endosc Surg. 2009;1(1):59-62.

2. Semmens J.P. Congenital anomalies of female genital tract. obstet. Gynecol. 1962;19:328-50.

3. Carinci F, Pezzetti F, Locci P, Becchetti E, Carls F, Avantaggiato A, et al. clinical findings, genes and extracellular matrix. J Craniofac Surg. 2005;16(3):361-8.

4. Simon C, Martinez L, Pardo F, et al. Mullerian defects in women with normal reproductive outcome. 1991;56:1192-3. 
5. Zurawin RK, Dietrich JE, Heard MJ, Edwards CL. Didelphic uterus and obstructed hemivagina with renal agenesis: case report and review of the literature. Journal of Pediatric and Adolescent Gynecology. 2004;17(2):137-41.

Cite this article as: Lavanya K., Cheruvu SC. A case report on uterus didelphys with unilateral renal agenesis and crouzon syndrome. Int $\mathbf{J}$ Reprod Contracept Obstet Gynecol 2015;4:1200-2. 